\title{
Optimal Blind Feedforward Carrier Synchronization for General QAM Modulations
}

\author{
Yan Wang and Erchin Serpedin \\ Dept. of Electrical Engineering \\ Texas A\&M University \\ College Station, TX 77843, USA
}

\author{
Philippe Ciblat \\ Ecole Nationale Supérieure des Télécomm. \\ Paris, France
}

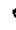

\section{Abstract}

This paper introduces a family of optimal blind feedforward estimators for joint estimation of the carrier phase and frequency offset of general quadrature amplitude modulated (QAM) transmissions. The asymptotic (large sample) performance of these estimators is established in closed-form expression. A practical implementation of the proposed optimally matched estimator, which is a computationally efficient approximation of the latter and exhibits negligible performance loss, is also derived. It is shown that the proposed family of constellation-dependent optimal estimators outperforms all the existing carrier synchronizers, and serves as an unifying framework for designing blind feedforward carrier recovery schemes for large $Q A M$ modulations. Finally, computer simulations are presented to show the merit of the proposed optimal estimators.
\end{abstract}

\section{Introduction}

Quadrature amplitude modulations (QAM) are currently used in throughput efficient high speed communication applications such as digital TV and TDMA systems. One of the main problems associated with the use of QAM modulations is that of carrier acquisition which for efficiency reasons must be performed without using preambles [1], [2], [3].

Carrier recovery involves the acquisition of both the carrier frequency and phase. Recently, assuming that the frequency recovery has already been achieved, a number of blind phase estimators for square and cross QAM modulations were reported in [1], [2], [3], [5, pp. 266-277] and [6]. These estimators exploit the angle information contained in the fourth or higherorder statistics of the received signal. However, none of the above estimators has been optimized, thus, they all exhibit rather poor performance (larger symbol error rate (SER)) at medium and high Signal-to-Noise Ratios (SNRs).
In this paper, a family of non-data aided (NDA) or blind feedforward joint carrier phase and frequency offset estimators for general QAM modulations is proposed and its asymptotic performance analyzed in a rigorous way. The proposed estimators represent a generalized form of the maximum likelihood feedforward algorithm, which was originally proposed by A. J. Viterbi and A. M. Viterbi as a blind carrier phase estimator for fully modulated M-PSK transmissions [9], [7]. An optimally "matched" estimator that achieves the smallest asymptotic variance within this family of blind estimators, together with a practical computationally efficient algorithm, is proposed and shown to improve the performance of the estimators proposed in the literature, especially in medium and high SNR ranges.

\section{Problem Formulation}

Let us consider the following baseband model:

$$
\begin{aligned}
& x(n)=w(n) e^{j \eta(n)}+v(n), \quad n=0, \ldots, N-1,(1) \\
& \eta(n):=\theta+2 \pi F_{e} T n
\end{aligned}
$$

where $\{w(n)\}$ is the independently and identically distributed (i.i.d.) input M-QAM symbol stream with zero-mean and unit variance $\left(\sigma_{w}^{2}:=\mathrm{E}\left\{|w(n)|^{2}\right\}=1\right)$, $T$ denotes the symbol period, $\{v(n)\}$ is a zero-mean circular white Gaussian noise process independent of $w(n)$ and with variance $\sigma_{v}^{2}:=\mathrm{E}\left\{|v(n)|^{2}\right\}, \theta$ and $f_{e}:=F_{e} T$ stand for the unknown carrier phase and frequency offset, respectively, which are the parameters to be estimated based only on knowledge of received samples $\{x(n)\}_{n=0}^{N \sim 1}$. The Signal-to-Noise Ratio per symbol is defined as SNR:=10 $\log _{10}\left(\sigma_{w}^{2} / \sigma_{v}^{2}\right)$.

Because the input QAM constellation has quadrant $(\pi / 2)$ symmetry, it follows that the estimates of $\theta$ and $f_{e}$ present 4-fold ambiguity, which can be counteracted by applying differential encoding. Without any loss of generality, we assume that the unknown phase $\theta$ lies in the interval $(-\pi / 4, \pi / 4)$ and $\left|f_{e}\right|<1 / 8$. 


\section{Estimators for QAM Constellations}

For convenience, we introduce a new notation $\mathcal{A}_{M}$ to denote the range of M-QAM constellations. With normalized energy, $w(n)$ takes a value from the set $\left(1 / r_{w}\right)\left\{ \pm(1+2 l) \pm j(1+2 k),(l, k) \in \mathcal{A}_{M}\right\}$, where $\mathcal{A}_{M}$ is defined for square QAM constellations (i.e., with sizes $\left.M=2^{2 m}, m=1,2, \ldots\right)$ and cross QAM constellations (i.e., with sizes $M=2^{2 m+1}, m=2,3, \ldots$ ) as $\mathcal{A}_{M}:=\left\{\left(0,1,2, \ldots, 2^{m-1}-1\right)^{2}\right\}$ and $\mathcal{A}_{M}:=$ $\left\{\left(0,1, \ldots, 3 \cdot 2^{m-2}-1\right)^{2}-\left(2^{m-1}, \ldots, 3 \cdot 2^{m-2}-1\right)^{2}\right\}$, respectively, and

$$
r_{w}^{2}:=\frac{4}{M} \sum_{(l, k) \in \mathcal{A}_{M}}\left[(1+2 l)^{2}+(1+2 k)^{2}\right]
$$

is an energy normalization constant.

Represent $x(n)$ in its polar form:

$$
x(n)=\rho(n) e^{j \phi(n)},
$$

and define the process $y(n)$ via the nonlinear transformation:

$$
y(n):=F(\rho(n)) e^{j 4 \phi(n)},
$$

where $F(\cdot)$ is a real-valued non-negative arbitrary nonlinear function. It is interesting to remark that the transformation (3) differs from the class of nonlinear transformations introduced in [7], [9]. This difference is due to the fact that all QAM constellations exhibit quadrant symmetries which translate into nonzero fourth-order moments $\left(\mathrm{E}\left\{w^{4}(n)\right\} \neq 0\right)$, and consequently justify the special form of the exponential factor in (3).

Conditioned on the signal $w(n), x(n)$ is normally distributed with the probability density function (pdf) $f(x(n) \mid w(n)) \sim \mathcal{N}\left(w(n) \exp (j \eta(n)), \quad \sigma_{v}^{2}\right)$. Throughout the paper, the notation $f(\cdot)$ will stand for the pdf of certain random variables (RVs). Due to $(2)$, it is easy to infer that the joint pdf of $\rho(n)$ and $\phi(n)$, and marginal pdf of $\rho(n)$ take the expressions:

$$
\begin{aligned}
& f(\rho(n), \phi(n))=\frac{\rho(n)}{M \pi \sigma_{v}^{2}} \sum_{(l, k) \in \mathcal{A}_{M}} \sum_{m=0}^{3} e^{-\frac{1}{\sigma_{v}^{2}}\left[\rho^{2}(n)+\varrho_{l, k}^{2}\right]} \\
& f(\rho(n))=\int_{-\pi}^{\pi} f(\rho(n), \phi(n)) \mathrm{d} \phi(n) \\
& =\frac{8 \rho(n)}{M \sigma_{v}^{2}} \sum_{(l, k) \in \mathcal{A}_{M}} e^{-\left(\rho^{2}(n)+\varrho_{l, k}^{2}\right) / \sigma_{v}^{2}} I_{0}\left(\frac{2 \rho(n) \varrho_{l, k}}{\sigma_{v}^{2}}\right),(5)
\end{aligned}
$$

where $\varrho_{l, k}:=\sqrt{\left[(1+2 l)^{2}+(1+2 k)^{2}\right]} / r_{w}, \psi_{l, k}:=$ $\arctan ((1+2 k) /(1+2 l))$, and $I_{0}(\cdot)$ stands for the zero- order modified Bessel function of the first kind. Moreover, since $w(n)$ and $v(n)$ are i.i.d. and mutually independent, based on (1) and (2), it is not difficult to find that the joint pdf of the RVs $\rho\left(n_{1}\right), \phi\left(n_{1}\right), \rho\left(n_{2}\right), \phi\left(n_{2}\right)$ satisfies the following factorization:

$$
\begin{aligned}
& f\left(\rho\left(n_{1}\right), \phi\left(n_{1}\right), \rho\left(n_{2}\right), \phi\left(n_{2}\right)\right) \\
& =f\left(\rho\left(n_{1}\right), \phi\left(n_{1}\right)\right) \cdot f\left(\rho\left(n_{2}\right), \phi\left(n_{2}\right)\right), \text { for } n_{1} \neq n_{2} .(6)
\end{aligned}
$$

Exploiting (4), some lengthy calculations lead to the following relations:

$$
\begin{aligned}
& \mathrm{E}\{y(n)\}=\mathrm{E}\left\{F(\rho(n)) e^{j 4 \phi(n)}\right\}=\mathcal{C} e^{j(\pi+4 \eta(n))} \\
& \mathcal{C}:=|\mathrm{E}\{y(n)\}|=\left|\mathrm{E}\left\{F(\rho(n)) e^{j 4 \phi(n)}\right\}\right|
\end{aligned}
$$

where the amplitude $\mathcal{C}$ is a real-valued constant which does not depend on $n$. From (6), it follows that $u(n):=y(n)-\mathrm{E}\{y(n)\}$ is wide sense stationary i.i.d., too. Consequently,

$$
y(n)=\mathcal{C} e^{j(\pi+4 \eta(n))}+u(n), n=0,1, \ldots, N-1 .
$$

Let $\boldsymbol{\omega}:=\left[\begin{array}{ll}\omega_{0} & \omega_{1}\end{array}\right]^{T}=\left[\begin{array}{ll}4 \theta & 8 \pi f_{e}\end{array}\right]^{T}$ and $\bar{\omega}$ be the trial value of $\omega$, and introduce the following NLS estimator (see e.g., [4]):

$$
\left\{\begin{array}{l}
\hat{\omega}_{1}=\arg \max _{\bar{\omega}_{1}} \frac{1}{N}\left|\sum_{n=0}^{N-1} y(n) e^{-j \bar{\omega}_{1} n}\right|^{2}, \\
\hat{\omega}_{0}=\operatorname{angle}\left\{-\sum_{n=0}^{N-1} y(n) e^{-j \hat{\omega}_{1} n}\right\} .
\end{array}\right.
$$

It is obvious that when $F(\rho(n))=\rho^{4}(n)$, the estimator (10) is just the standard fourth-power estimator $[5$, pp. 281-282], [6].

The asymptotic variance of estimator (10) is established in the following theorem:

Theorem 1 The asymptotic variance of the estimates $\hat{\omega}_{l}, l=0,1$ in (10) is given by:

$$
\begin{aligned}
& \operatorname{avar}\left(\hat{\omega}_{l}\right):=\lim _{N \rightarrow \infty} \mathrm{E}\left\{\left(\hat{\omega}_{l}-\omega_{l}\right)^{2}\right\}=\frac{\mathcal{B}-\mathcal{D}}{\mathcal{C}^{2}} \cdot \frac{4 l+2}{N^{2 l+1}}, \\
& \mathcal{B}:=\mathrm{E}\left\{|y(n)|^{2}\right\}=\mathrm{E}\left\{F^{2}(\rho(n))\right\} \\
& \mathcal{D}:=\left|\mathrm{E}\left\{y^{2}(n)\right\}\right|=\left|\mathrm{E}\left\{F^{2}(\rho(n)) e^{j 8 \phi(n)}\right\}\right|,
\end{aligned}
$$

and $\mathcal{C}$ is defined in (8).

Some calculations show that $\mathcal{B}, \mathcal{C}, \mathcal{D}$ take the following expressions:

$$
\begin{aligned}
& \mathcal{B}=\int_{0}^{\infty} F^{2}(\rho(n)) \xi_{1}(\rho(n)) \mathrm{d} \rho(n), \\
& \mathcal{C}=\int_{0}^{\infty} F(\rho(n)) \xi_{2}(\rho(n)) \mathrm{d} \rho(n), \\
& \mathcal{D}=\int_{0}^{\infty} F^{2}(\rho(n)) \xi_{3}(\rho(n)) \mathrm{d} \rho(n),
\end{aligned}
$$


where for $i=1,2,3$ the following relations hold:

$$
\begin{gathered}
\xi_{i}(\rho(n)):=(-1)^{i-1} \frac{8 \rho(n)}{M \sigma_{v}^{2}} e^{-\frac{\rho^{2}(n)}{\sigma_{v}^{2}}} \\
\cdot \sum_{l, k \in \mathcal{A}_{M}} \cos \left(4(i-1) \varphi_{l, k}\right) e^{-\frac{\rho_{l, k}^{2}}{\sigma_{v}^{2}}} I_{4(i-1)}\left(\frac{2 \rho(n) \varrho_{l, k}}{\sigma_{v}^{2}}\right)
\end{gathered}
$$

and $\varphi_{l, k}:=\psi_{\max \{l, k\}, \min \{l, k\}}$.

It is of interest to compare the asymptotic variance (11) with the Cramér-Rao lower bound (CRB). In [8], the CRBs for carrier phase and frequency offset estimates are derived for fully QAM-modulated carriers, and with the notations adopted so far admit the following expression for large $N$ :

$\operatorname{CRB}\left(\hat{\omega}_{l}\right)=\mathrm{CRB}_{\mathrm{CW}}(\hat{\theta}) \cdot R\left(\sigma_{v}^{2}\right)=\frac{(4 l+2) \sigma_{v}^{2}}{N^{2 l+1}} \cdot R\left(\sigma_{v}^{2}\right)$,

where $\mathrm{CRB}_{\mathrm{CW}}$ corresponds to the CRB for an unmodulated carrier wave, and $R\left(\sigma_{v}^{2}\right)$ denotes the constellation-dependent ratio of the true CRB to $\mathrm{CRB}_{\mathrm{CW}}$, which can be evaluated by means of numerical integration or Monte Carlo evaluations (MCE) [8]. Based on (11) and the expression of CRB, one can observe that the asymptotic variances of the estimator (10) decay at the same rate as the CRB.

In the absence of frequency offset $\left(f_{e}\right)$, the proposed NLS estimator (10) reduces to the phase estimator:

$$
\text { - } \hat{\theta}=\frac{1}{4} \text { angle }\left\{-\sum_{n=0}^{N-1} y(n)\right\},
$$

whose asymptotic variance is one quarter of that corresponding to the case of joint phase and frequency offset estimation [8], and is given by:

$$
\operatorname{avar}(\hat{\theta})=\frac{\mathcal{B}-\mathcal{D}}{32 N \mathcal{C}^{2}} .
$$

Next, we determine the optimal "matched" nonlinearity $F(\cdot)$ which minimizes the asymptotic variances. Since in (11), only the terms $\mathcal{B}, \mathcal{C}, \mathcal{D}$ depend on $F(\cdot)$, finding an optimal $F(\cdot)$ resorts to solving the optimization problem:

$$
F_{\min }(\rho(n))=\arg \min _{F(\cdot)} \frac{\mathcal{B}-\mathcal{D}}{\mathcal{C}^{2}} .
$$

Based on (14)-(16), the optimum nonlinearity $F_{\min }$ is obtained by using Cauchy-Schwarz' inequality and is given by the following theorem:

Theorem 2 The optimal "matched" nonlinearity $F_{\min }(\cdot)$ that minimizes the asymptotic variance of the proposed family of estimators (10) is given by:

$$
F_{\min }(\rho(n))=\lambda \frac{\xi_{2}(\rho(n))}{\xi_{1}(\rho(n))-\xi_{3}(\rho(n))},
$$

where $\lambda$ is an arbitrary nonzero constant selected such that $F_{\min }(\cdot)$ is non-negative.

Plugging (20) back into (14)-(16), and substituting these values into (11), the asymptotic variance corresponding to the optimal matched estimates $\hat{\omega}_{l}$, can be expressed as:

$$
\operatorname{avar}_{\text {min }}\left(\hat{\omega}_{l}\right)=\frac{4 l+2}{N^{2 l+1}} \cdot \frac{1}{\int_{0}^{\infty} \frac{\xi_{2}^{2}(\rho(n))}{\xi_{1}(\rho(n))-\xi_{3}(\rho(n))} \mathrm{d} \rho(n)} .
$$

In Fig. 1, assuming the number of samples $N=500$, we evaluate the theoretical asymptotic variances of the proposed optimal matched and fourth-power estimators versus SNR for 16-QAM. Since the difference between the asymptotic variances of $\hat{\theta}$ and $\hat{f}_{e}$ is just a constant for a given SNR, only the variance of $\hat{\theta}$ (19) is plotted. From Fig. 1, one can observe that at low SNRs, both the optimal estimator and the fourthpower estimator achieve CRB, which means that at very low SNRs, the classic fourth-power estimator is always the best choice. This is not a surprising result since the fourth-power estimator is simply a low-SNR approximation of the ML estimator [6]. However, in the more practical regime of medium and high SNRs, the optimum nonlinear estimator provides a significant improvement over the fourth-power estimator while the latter exhibits the error floor due to its self-induced noise [6], [8].

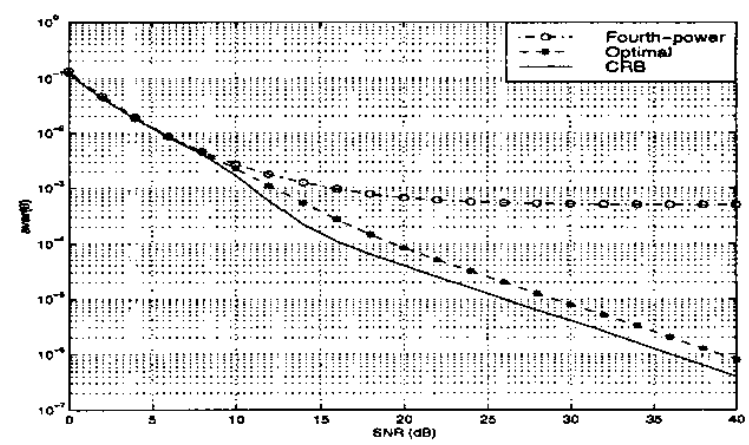

Figure 1: Theoretical bounds of $\hat{\theta}$ versus SNR (16QAM constellation).

\section{Implementation of the Optimal Esti- mator}

The result shown in Fig. 1 illustrates the good property of the optimal nonlinearity (20) for higher-order 
QAM modulations at medium and high SNR ranges. As can be observed from $(17)$ and $(20), F_{\min }(\rho(n))$ is a function that depends on the SNR, and presents high implementation complexity, which makes the optimal estimator impractical. Fortunately, computer simulations indicate that the sensitivity of the optimal estimator to SNR is limited in medium and high SNR ranges. By considering approximations of (20), we propose next computationally efficient SNRindependent estimators, which will be referred to as APP-estimators.

We select 16-QAM as an example to illustrate the derivation of the constellation-dependent APP estimator. Fig. 2 plots the optimal nonlinearity (20) versus the magnitude $\rho$ of the received data at $\mathrm{SNR}=20 \mathrm{~dB}$ for 16-QAM modulation. The curve presented in Fig. 2 suggests that for 16-QAM a good design for the APP estimator is a piecewise linear approximation of the following form:

$$
F_{\mathrm{APP}_{16}}(\rho(n))= \begin{cases}122.2733 \rho(n) & \text { if } \rho(n) \leq 0.7 \\ 331.885 \rho(n)-30.4524 & \text { if } \rho(n) \geq 1.2 \\ 0 & \text { elsewhere }\end{cases}
$$

Since $F_{\mathrm{APP}}(\cdot)$ is constellation-dependent, we will not present the detailed expressions of $F_{\mathrm{APP}}$ for other QAM modulations in this paper. The APP nonlinearities for general QAM constellations can be obtained in a similar way. Careful examination of the expressions of APP nonlinearities illustrates that the intrinsic principle of APP estimators is to emphasize the weight of the points located on the diagonals of the signal constellation, and discard all the off-diagonal points. It appears also that only a subset of the points located on the diagonals is selected.

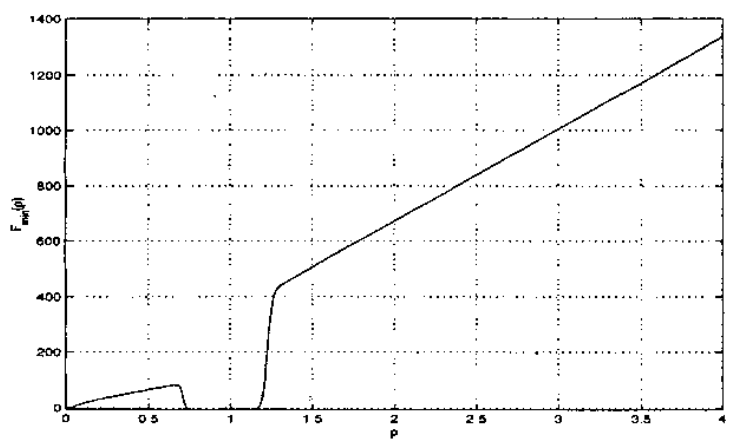

Figure 2: $F_{\min }$ versus $\rho$ (16-QAM constellation at $\mathrm{SNR}=20 \mathrm{~dB})$.

\section{Simulation Experiments}

In this section, we study thoroughly the performance of estimators (10) and (18) using computer sim- ulations. The experimental mean-square error (MSE) results of the proposed estimators will be compared with the theoretical asymptotic bounds and the CRB. The impact of the nonlinearity $F(\cdot)$ on SER is also assessed. The additive noise is generated as zeromean Gaussian white noise, the carrier phase $\theta=0.2$ and frequency offset $F_{e} T=0.05$, the number of samples is assumed $N=500$, and the experimental results are obtained by performing a large number of $M C=100,000$ Monte Carlo trials to ensure accuracy. Experiment 1-Comparison of the MSE of the proposed estimators with the theoretical bounds versus SNR: This experiment compares the theoretical (The.) bounds with the experimental (Exp.) MSEs of the proposed estimators (10) for 16-QAM (Figs. 3 and 4). These figures show that for medium and high SNRs, the experimental results of the optimal estimator and the fourth-power estimator are well predicted by the asymptotic bounds derived in this paper, and the proposed optimal estimator provides considerable improvement over the fourth-power estimator.

Experiment 2-The impact of the nonlinearity on SER: In Fig. 5, we show the SER performance of the carrier synchronizer (18) exploiting different nonlinearities for 32-QAM. We also plot the result of the eighth-order statistics based phase estimator (EOE) proposed for cross QAM in [1]. To show the superior performance of the optimal estimator, we also plot as a lower bound the SER curves in the case of perfect carrier recovery, i.e., in the case when the transmitted symbols are only corrupted by additive white Gaussian noise (AWGN). Fig. 5 indicates that the optimal estimator approaches closely this lower bound, and improves significantly the performance of the conventional fourth-power estimator and EOE for medium and high SNRs. We can also observe that APP is a satisfying realizable alternative to the optimal estimator.

Experiment 3-The performance of the proposed estimators in the case of higher-order QAM modulations: Fig. 6 illustrates the performance of the optimal estimator and APP for larger-order QAM modulation (256-QAM), compared with the fourth-power estimator. Since higher-order QAM modulations often operate at larger SNRs, we pay special attention to the medium and high SNRs, where the SER is in the range $\mathrm{SER} \leq 10^{-3}$. This figure shows again the merit of the proposed optimal estimator and APP.

\section{Conclusions}

In this paper, we have introduced and analyzed a family of blind feedforward carrier synchronizers for general QAM modulations. Based on a generalization of the $\mathrm{V} \& \mathrm{~V}$ algorithm, an optimal matched nonlin- 
ear estimator is introduced and its performance established in closed-form expressions. A framework for designing computationally efficient approximations of the proposed optimal estimator without incurring much performance loss, is also proposed. Simulation results indicate the superior performance of the proposed (approximate) optimal estimator compared with the existing methods, and the merit of the performance analysis presented in this paper.

\section{Acknowledgments}

This work was supported by the NSF Career Award No. CCR-0092901.

\section{References}

[1] K. V. Cartwright, "Blind phase recovery in cross QAM communication systems with eighth-order statistics," IEEE Signal Proc. Letters, vol. 8, pp. 304-306, Dec. 2001.

[2] L. Chen, H. Kusaka and M. Kominami, "Blind phase recovery in QAM communication systems using higher order statistics," IEEE Signal Proc. Letters, vol. 3, pp. 147-149, May 1996.

[3] C. N. Georghiades, "Blind carrier phase acquisition for QAM constellations," IEEE Trans. Commun., vol. 45, pp. 1477-1486, Nov. 1997.

[4] M. Ghogho and A. Swami, "Non-efficiency of the nonlinear least-squares estimator of polynomial phase signals in colored noise," Conf. Rec., Asilomar'98, pp. 1447-1451, Pacific Grove, 1998.

[5] U. Mengali and A. N. D' Andrea, Synchronization Techniques for Digital Receivers, Plenum Press, New York, 1997.

[6] M. Moeneclaey and G. de Jonghe, "ML-oriented NDA carrier synchronization for general rotationally symmetric signal constellations," IEEE Trans. Commun., vol. 42, no. 8, pp. 2531-2533, Aug. 1994.

[7] B. E. Paden, "A matched nonlinearity for phase estimation of a PSK-modulated carrier," IEEE Trans. Inform. Theory, vol. 32, pp. 419-422, May 1986.

[8] F. Rice, B. Cowley, B. Moran and M. Rice, "Cramér-Rao lower bound for QAM phase and frequency estimation," IEEE Trans. Commun., vol. 49, no. 9, pp. 1582-1591, Sept. 2001.

[9] A. J. Viterbi and A. M. Viterbi, "Nonlinear estimation of PSK-modulated carrier phase with application to burst digital transmissions," IEEE Trans. Inform. Theory, vol. 29, pp. 543-551, July 1983.

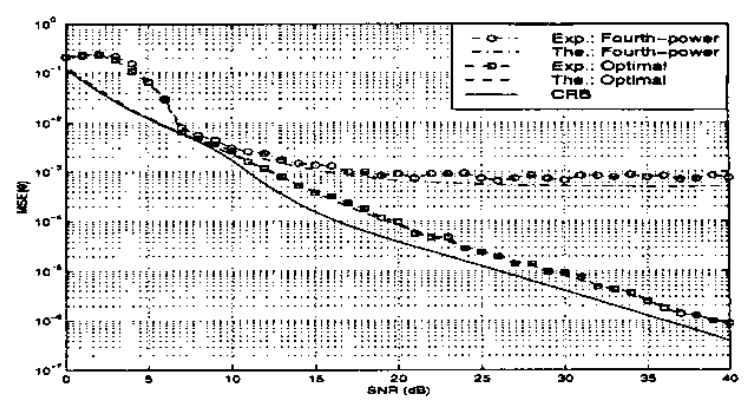

Figure 3: Comparison of MSEs of $\hat{\theta}$ versus SNR (16QAM).

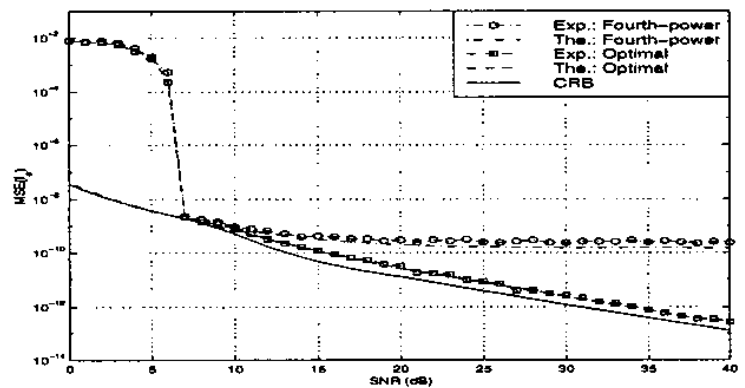

Figure 4: Comparison of MSEs of $\hat{f}_{e}$ versus SNR (16QAM).

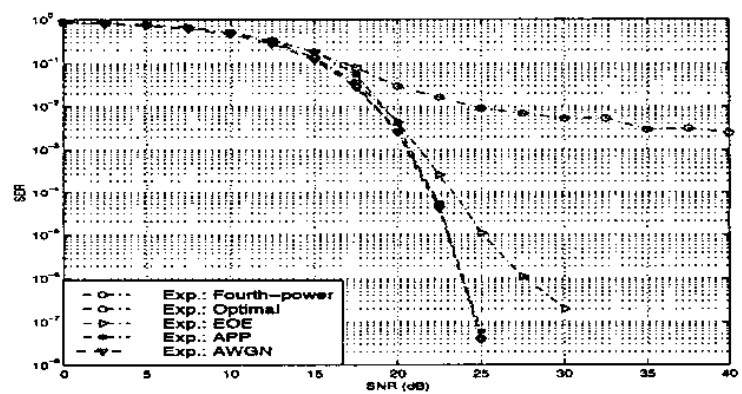

Figure 5: SER curves versus SNR (32-QAM).

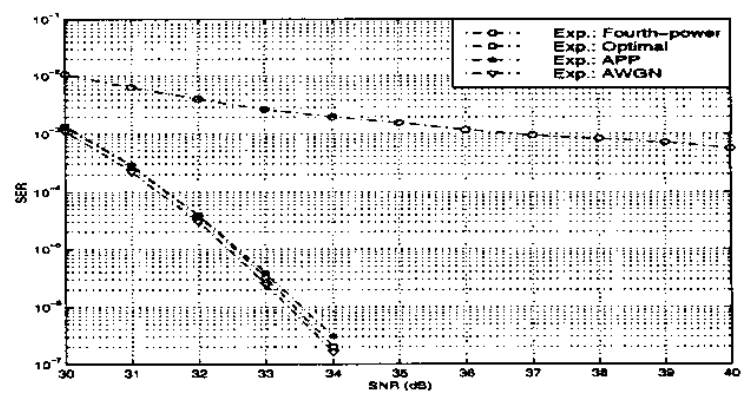

Figure 6: SER curves versus SNR (256-QAM). 